TITLE:

\title{
Time-domain response of point- defect cavities in two-dimensional photonic crystal slabs using picosecond light pulse
}

$\operatorname{AUTHOR}(S)$ :

Asano, T; Kunishi, W; Song, BS; Noda, S

\section{CITATION:}

Asano, T ... [et al]. Time-domain response of point-defect cavities in two-dimensional photonic crystal slabs using picosecond light pulse. APPLIED PHYSICS LETTERS 2006, 88(15): 151102.

\section{ISSUE DATE:}

2006-04-10

URL:

http://hdl.handle.net/2433/50165

\section{RIGHT:}

Copyright 2006 American Institute of Physics. This article may be downloaded for personal use only. Any other use requires prior permission of the author and the American Institute of Physics. 


\title{
Time-domain response of point-defect cavities in two-dimensional photonic crystal slabs using picosecond light pulse
}

\author{
Takashi Asano, ${ }^{\text {a) }}$ Wataru Kunishi, Bong-Shik Song, and Susumu Noda ${ }^{\text {b) }}$ \\ Department of Electronic Science and Engineering, Kyoto University, Kyoto 615-8510, Japan
}

(Received 24 October 2005; accepted 2 March 2006; published online 10 April 2006)

\begin{abstract}
Time-domain responses of point-defect cavities in two-dimensional photonic crystal slabs are directly investigated. Point-defect cavities are excited by picosecond light pulses and time evolutions of the light emissions are measured by using cross-correlation method. The obtained signals clearly show delays in initial rises and tailings in later stages which reflect photon lifetimes of the cavities. The signals are analyzed by comparing with numerical simulations where Gaussian input pulses and linear responses of the systems are assumed. Photon lifetimes are evaluated to be $<1,3$, and 9 ps for the point-defect cavities having $Q$ factors of 500, 3800, and 10000 , respectively. We believe that the results obtained here will contribute to the time-domain manipulation of photons utilizing artificial defects in photonic crystals. () 2006 American Institute of Physics.
\end{abstract}

[DOI: $10.1063 / 1.2194010]$

Photonic $\mathrm{crystal}^{1,2}(\mathrm{PC})$ is an optical material with a periodic refractive index change, where a frequency gap called as photonic band gap (PBG) is created in the photonic mode spectrum. Recently, a thin slab of two-dimensional PC (2D-PC slab) $)^{3-7}$ is drawing much attention because they can control photons strongly in spite of relatively easy fabrication process of 2D structure. High performance photonic cavities, which have small cavity volumes of the cubic wavelength order and high $Q$ factors of the order of $10^{3}-10^{6}$, can be created by introducing carefully designed point defects in 2D PC slabs. ${ }^{8-14}$ Various useful characteristics, such as high resolution with large free spectral range, ${ }^{8,14}$ feasibility for integrated optical system, ${ }^{7,15-17}$ and increased light-matter interactions $^{18-23}$ are expected in such cavities. Moreover, they must have long photon lifetimes of several to several hundreds of picoseconds, and the photon lifetimes may be improved up to nanosecond region in near future. Since the point-defect cavities in 2D-PC slabs have the ability to storage photons together with the feasibility of integration, they are expected to be applied to ultrasmall devices to control light pulses or photons in time domain ${ }^{24,25}$ (optical buffer memories, quantum information devices, etc.) Thus, it is considered very interesting to investigate the responses of such ultrasmall and high- $Q$ cavities directly in the time domain. Although time-domain measurements on bulk PC (Refs. 26-28) and line-defect waveguide in PC slabs ${ }^{29-31}$ have been already reported, there are no reports on defect cavities. In this report, we show the measurement results where the point-defect cavities in 2D-PC slabs are excited by picosecond light pulses and the evolutions of the light emission from the cavities are measured by optical crosscorrelation method. Photon lifetime of point-defect cavities having $Q$ factors from $10^{2}-10^{4}$ are measured and discussed.

Samples investigated were air-bridge-type 2D-PC slabs consisting of $\mathrm{Si}$ which have triangular lattice patterns of round air holes. The lattice constants $(a)$ of PCs are $405-420 \mathrm{~nm}$, and the radii of air holes are $120 \mathrm{~nm}$. Each sample has a point-defect cavity and a line-defect waveguide through which the cavity is excited. Silicon on insulator

\footnotetext{
${ }^{a)}$ Electronic mail: tasano@kuee.kyoto-u.ac.jp

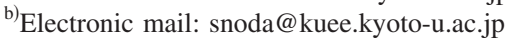

(SOI) substrates, which consist of a 250-nm-thick Si layer, 1.5- $\mu$ m-thick $\mathrm{SiO}_{2}$ layer, and 700- $\mu$ m-thick $\mathrm{Si}$ layer, were used for the fabrication. First, the PC patterns were formed on the top Si layers by using electron beam lithography and induction coupled plasma etching techniques. Next, the $\mathrm{SiO}_{2}$ layers underneath the PC patterns were removed by selective wet etching to form air-bridge-type slabs. Three different samples shown in the insets of Figs. 1(a)-1(c) were prepared. The point defect in sample A is an air hole which is larger than regular lattice points. ${ }^{7,32}$ The point defect in sample B are three missing air holes aligned along $\Gamma-J$ direction. The point defect in sample $\mathrm{C}$ is almost the same as that in sample B except that two air holes at both edges of the defect are shifted by $0.1 a$ toward outside in order to increase the $Q$ factor. $^{8}$ The lattice constants of these samples are designed ${ }^{15}$ so that the resonant wavelengths of the cavities are within the tunable range of the light source $(1530-1555 \mathrm{~nm})$ used for the experiments. Also, the widths of the line-defect waveguides $(W)$ are designed so that their transmitting ranges cover the resonant wavelengths of the cavities. The distances between the cavities and waveguides $(D)$ are designed so that light emission efficiencies from the cavities to free space become maximal under $\mathrm{cw}$ excitation condition. ${ }^{32}$ Resonant spectra of the samples A-C are shown in Figs. $1(\mathrm{a})-1(\mathrm{c})$, respectively, where the cavities were excited by $\mathrm{cw}$ light through the waveguides, and emission intensities from the cavities to free space were measured as functions of excitation wavelength. The $Q$ factors of samples A, B, and C are evaluated to be 500,3800 , and 10000 , respectively, from the resonant spectra. The oscillation pattern seen in Fig. 1(a) is an artifact induced by the reflections at the both edges of the waveguide (Fabry-Pérot interference). The samples B and $\mathrm{C}$ do not show such oscillation patterns since they have antireflection structures, where slabs without PC patterns are extended after the output ends of the PC waveguides [see inset of Fig. 1(c)].

Figure 2 schematically shows the experimental setup used for time-domain measurements. A passive mode-lock fiber laser was used as a light source, where the center wavelength $\left(\lambda_{c}\right)$ of light pulses is tunable between 1530 and $1555 \mathrm{~nm}$, and the duration [full width at half maximum (FWHM)] of light pulses is semitunable between 3 and 5 ps. 
(a)
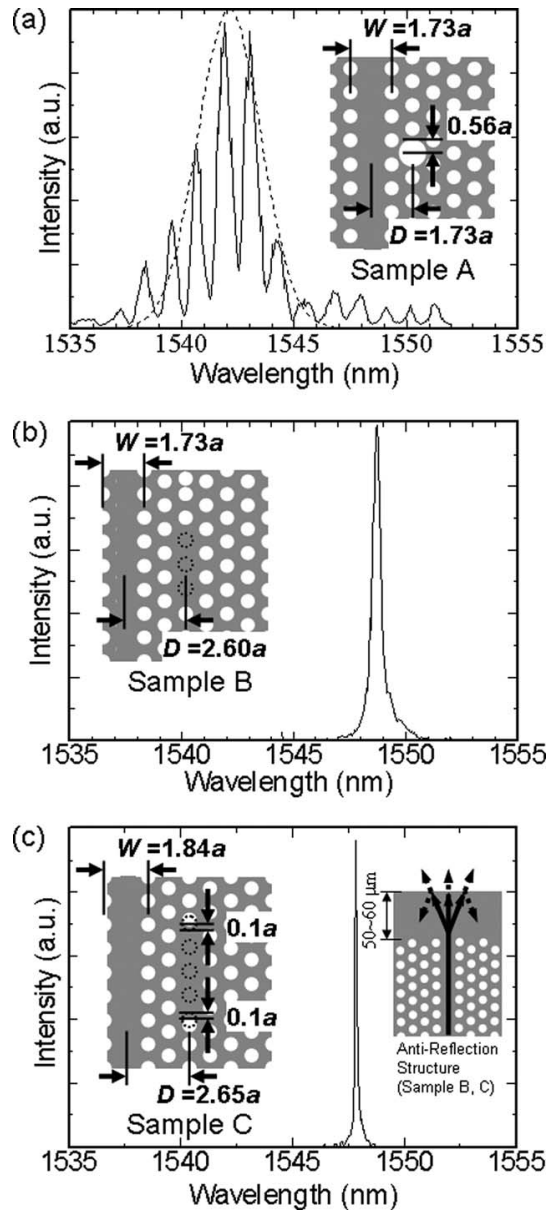

FIG. 1. Resonant spectra of defect cavities obtained in wavelength-domain measurements; (a) acceptor-type defect with an enlarged air hole (dashed line is a guide to eyes), (b) donor-type defect with three missing air holes, and (c) donor-type defect with three missing air holes and air hole shifts. Insets: schematics showing the structures of the samples.

After $\lambda_{c}$ was tuned to the resonant peak of a cavity, the light pulse was incident to the waveguide from the input facet to excite the cavity. The energy of the light pulse incident into the waveguide was estimated to be smaller than $1 \mathrm{pJ}$. The light emitted from the cavity was collected by an objective lens, was relayed to a single mode fiber (using path A shown in Fig. 2), and was amplified by an Er doped optical fiber amplifier. Another light pulse was separated directly from the light source and was utilized as a reference pulse. Crosscorrelation signal between the reference light pulse and the (amplified) cavity emission was measured by using a second harmonic generation and delay scanning methods. The responses of the cavities were evaluated by comparing the signals with and without samples. (The latter signals were measured by using path B shown in Fig. 2.)

Figure 3(a) shows the cross-correlation signal obtained with sample A (open circle) and that obtained without samples (open squares). It is seen in the figure that both signals have almost same shapes except that the signal with the sample has another small peak at a delay time of $\sim 9$ ps. The subsequent peak is not attributed to the response of the cavity itself. It is the light which was not trapped by the cavity at first and experienced an additional round trip along the waveguide ${ }^{30}$ to be trapped by the cavity for the second time. This phenomenon is consistent with the fact that FabryPérot interference pattern is clearly observed in the resonant spectrum [Fig. 1(a)]. As will be shown below, such subseDownloaded 05 Mar 2008 to 130.54 .110 .22 . Redistribution subject

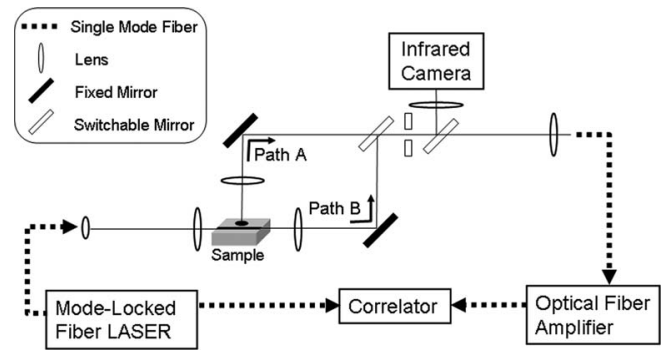

FIG. 2. Schematic diagram of the measurement setup utilized for the timedomain experiment.

quent peaks are not observed for samples $\mathrm{B}$ and $\mathrm{C}$ because the reflections at the facets are reduced by modifying the facet structure as described before. Concerning with the main peaks, the correspondence of the signals with and without the sample indicates the fact that the photon lifetime in the point-defect cavity is much shorter than the duration of the signal without samples (FWHM $\sim 6 \mathrm{ps}$ ). This is consistent with the $Q$ factor $(\sim 500)$ obtained from the wavelengthdomain measurement [Fig. 1(a)], from which the photon lifetime is estimated to be about 0.4 ps.

Figures 3(b) and 3(c) show the results of the crosscorrelation measurement for samples $\mathrm{B}$ and $\mathrm{C}$, respectively. It is seen in Fig. 3(b) that the signal with a sample (open circle) has a slowly decaying tail while that without samples (open square) has a symmetric wave form. FWHM of the former (7.6 ps) is about 1.5 times of that of the latter (5.2 ps). For sample C, FWHM of the signal with the sample (14 ps) is almost two times of that without samples (6.8 ps). The decaying tails and broadenings of the signals are considered to reflect the fact that photons are trapped in the cavities for certain lengths of time before emitted to free space. Here, it is worth to mention that such tailings and broadenings were not observed for the pulses which transmit only through the line-defect waveguides without being trapped by the cavity, where $\lambda_{c}$ is slightly detuned from the cavity resonance and the light pulses emitted from the other edge of the waveguide were observed by using path B.

Next, we quantitatively analyzed the cross-correlation signals to obtain photon lifetimes in the cavities. The crosscorrelation signal without a sample $I(t)$ can be expressed as

$$
I(t)=\int_{-\infty}^{+\infty}\left|i\left(t^{\prime}\right) * m\left(t^{\prime}\right)\right|^{2}\left|r\left(t^{\prime}-t\right)\right|^{2} d t^{\prime},
$$

where $i(t)$ is the electric field envelope of an input light pulse, $r(t)$ is that of a reference light pulse, $m(t)$ is the impulse response of the measurement system, and the asterisk represents a convolution operator. The impulse response of the cavity having a photon lifetime $\tau$ can be expressed as $\exp (-t / 2 \tau)$. Therefore, the cross-correlation signal with a sample $[O(t)]$ is expressed as

$$
O(t)=\int_{-\infty}^{+\infty}\left|\left\{i\left(t^{\prime}\right) * m\left(t^{\prime}\right)\right\} * \exp \left(-t^{\prime} / 2 \tau\right)\right|^{2}\left|r\left(t^{\prime}-t\right)\right|^{2} d t^{\prime} .
$$

The fittings of measurement results were carried out as follows. At first, the wave form of $i(t) * m(t)$ was determined by fitting the cross-correlation signal without a sample using Eq. (1). Here, it was assumed that wave forms of $i(t) * m(t)$ and $r(t)$ were Gaussian functions. In addition, the width of $r(t)$ was determined beforehand by measuring a correlation signal between the reference pulses themselves. Next, the AlP license or copyright; see http://apl.aip.org/apl/copyright.jsp 
(a)

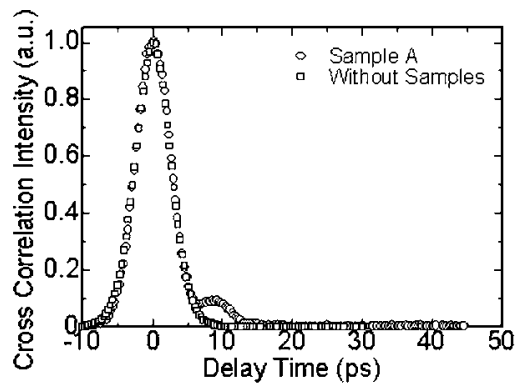

(b)

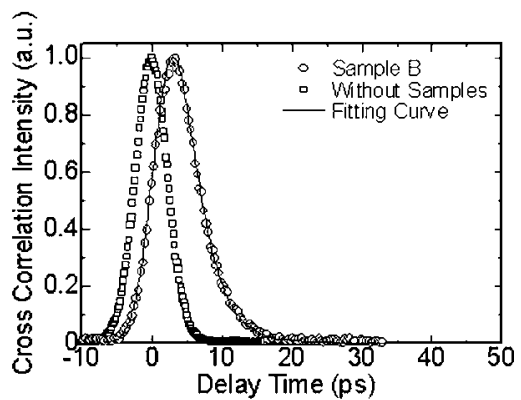

(c)

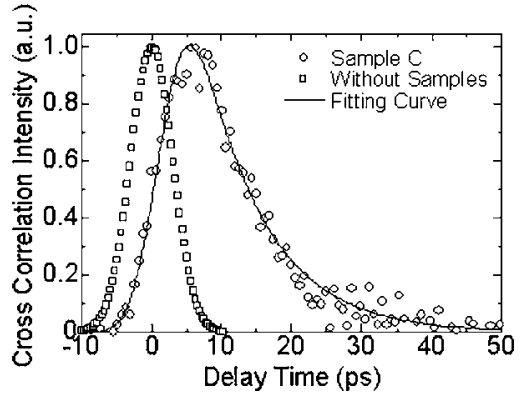

FIG. 3. Cross-correlation signal obtained with (open circle) and without (open square) samples; (a) acceptor-type defect with an enlarged air hole, (b) donor-type defect with three missing air holes, and (c) donor-type defect with three missing air holes and air hole shifts. Solid lines in (b) and (c) are theoretical fitting curves assuming photon lifetimes of 3 and $9 \mathrm{ps,}$ respectively.

cross-correlation signal with a sample was fitted by using Eq. (2) to obtain $\tau$. In this fitting, the relative time positions between the incident light $I(t)$ and emitted light $O(t)$ are also obtained.

The best fitting results are shown in Figs. 3(b) and 3(c) by solid lines. Photon lifetimes obtained for samples B and C were 3.0 and $9.0 \mathrm{ps}$, respectively. The photon lifetimes of ultrasmall defect cavities with cubic wavelength size have been successfully measured in the time domain. In addition, the increase of a photon lifetime due to the shift of air holes (sample C) has been clearly demonstrated. The lifetimes obtained in the time-domain experiments were almost consistent with that evaluated from the wavelength-domain measurements (3.1 ps for sample B and 8.2 ps for sample C). This agreement indicates that the cavities investigated here exhibit almost linear responses. Nonlinear responses of PC cavities were observed for more intense input light, and such results will be reported separately. Concerning with the peak positions of the signals, the delays between the incident and emitted lights are observed in Figs. 3(b) and 3(c). The intensities of the emitted light are proportional to the light energy stored in the cavities. Since the response of the cavities is slower than the pulse widths, the cavities operate like integrator so that the stored energy becomes maximal when the incident pulses have almost passed by the cavities. Therefore, the peak position of the emitted light delays compared to the incident light pulse by about the duration of the incident light pulse.

In summary, we have succeeded in measuring the timedomain response of the point-defect cavities in 2D-PC slab by using picosecond light pulses. Photon lifetimes of the resonant modes of the cavities are evaluated by analyzing the obtained cross-correlation wave forms. A photon lifetime of $9 \mathrm{ps}$ is observed for a cubic wavelength size cavity having a $Q$ factor of 10000 . We believe that the results obtained here will contribute to the time-domain manipulation of photons utilizing artificial defects in PCs.

This work is partly supported by Core Research for Evolution Science and Technology (CREST), Japan Science and Technology Corporation (JST), and Grant-in-Aids for Scientific Research from the Ministry of Education, Culture, Sport, Science, and Technology of Japan.

${ }^{1}$ E. Yablonovich, Phys. Rev. Lett. 58, 2059 (1987).

${ }^{2}$ S. Noda, K. Tomoda, N. Yamamoto, and A. Chutinan, Science 289, 605 (2000).

${ }^{3}$ A. Chutinan and S. Noda, Jpn. J. Appl. Phys., Part 1 39, 2353 (2000).

${ }^{4}$ A. Chutinan and S. Noda, Phys. Rev. B 62, 4488 (2000).

${ }^{5}$ S. G. Jonson, P. R. Villeneuve, S. Fan, and J. D. Joannopoulos, Phys. Rev. B 62, 8212 (2000).

${ }^{6}$ O. Painter, J. Vuckovic, and A. Scherer, J. Opt. Soc. Am. B 16, 275 (1999).

${ }^{7}$ S. Noda, A. Chutinan, and M. Imada, Nature (London) 407, 608 (2000).

${ }^{8}$ Y. Akahane, T. Asano, B. S. Song, and S. Noda, Nature (London) 425 , 944 (2003).

${ }^{9}$ K. Srinivasan, P. E. Barclay, O. Painter, J. Chen, A. Y. Cho, and C. Gmachl, Appl. Phys. Lett. 83, 1915 (2003).

${ }^{10}$ H. Y. Ryu, M. Notomi, and Y. H. Lee, Appl. Phys. Lett. 83, 4294 (2003)

${ }^{11}$ T. Asano and S. Noda, Nature (London) 429, 6988 (2004).

${ }^{12}$ K. Srinivasan, P. E. Barclay, and O. Painter, Opt. Express 12, 1458 (2004)

${ }^{13}$ Y. Akahane, T. Asano, B. S. Song, and S. Noda, Opt. Express 13, 1202 (2005).

${ }^{14}$ B. S. Song, S. Noda, T. Asano, and Y. Akahane, Nat. Mater. 4, 207 (2005).

${ }^{15}$ B. S. Song, S. Noda, and T. Asano, Science 300, 1537 (2003).

${ }^{16}$ B. S. Song, T. Asano, Y. Akahane, Y. Tanaka, and S. Noda, J. Lightwave Technol. 23, 1449 (2005).

${ }^{17}$ H. Takano, B. S. Song, T. Asano, and S. Noda, Appl. Phys. Lett. 86, 241101-1 (2005).

${ }^{18}$ M. Loncar, A. Scherer, and Y. Qiu, Appl. Phys. Lett. 82, 4648 (2003).

${ }^{19}$ T. Uesugi, B. S. Song, T. Asano, and S. Noda, Opt. Express 14, 377 (2006).

${ }^{20}$ T. Asano, W. Kunishi, M. Nakamura, B. S. Song, and S. Noda, Extended Abstract of 65th Meeting of Japan Society of Applied Physics, 2004 (unpublished), p. 942.

${ }^{21}$ P. E. Barclay, K. Srinivasan, and O. Painter, Opt. Express 13, 801 (2005).

${ }^{22}$ M. Notomi, A. Shinya, S. Mitsugi, G. Kira, E. Kuramochi, and T. Tanabe, Opt. Express 13, 2678 (2005).

${ }^{23}$ T. Yoshie, A. Scherer, J. Hendrickson, G. Khitrova, H. M. Gibbs, G. Rupper, C. Ell, O. B. Shchekin, and D. G. Deppe, Nature (London) 432, 200 (2004).

${ }^{24}$ M. F. Yanik and S. Fan, Phys. Rev. Lett. 92, 083901 (2004).

${ }^{25}$ Y. Tanaka, T. Asano, and S. Noda, Technical Digest of ICQE/CLEO-PR, CWE4-3, 2005 (unpublished).

${ }^{26}$ T. Tanaka, S. Noda, A. Chutinan, T. Asano, and N. Yamamoto, Opt. Quantum Electron. 34, 37 (2002).

${ }^{27}$ K. Inoue, N. Kawai, Y. Sugimoto, N. Charlsson, N. Ikeda, and K. Asakawa, Phys. Rev. B 65, 121308(R) (2002).

${ }^{28}$ M. C. Netti, C. E. Finlayson, J. J. Baumberg, M. D. B. Charlton, M. E. Zoorob, J. S. Wilkinson, and G. Parker, Appl. Phys. Lett. 81, 3927 (2002).

${ }^{29}$ H. Gersen, J. P. Korterik, N. F. van Hulst, and L. Kuipers, Phys. Rev. 68 , 026604 (2003).

${ }^{30}$ T. Asano, K. Kiyota, D. Kumamoto, B. S. Song, and S. Noda, Appl. Phys. Lett. 84, 4690 (2004).

${ }^{31}$ R. J. P. Engelen, T. J. Karle, H. Gersen, J. P. Korterik, T. F. Krauss, L. Kuipers, and N. F. van Hulst, Opt. Express 13, 4457 (2005).

${ }^{32}$ A. Chutinan, M. Mochizuki, M. Imada, and S. Noda, Appl. Phys. Lett. 79 2690 (2001) 